\title{
On the correspondence between variational principles in Eulerian and Lagrangian descriptions
}

\author{
Alexander V. Aksenov ${ }^{*, * * 1}$ and Konstantin P. Druzhkov,***,2 \\ ${ }^{*}$ Lomonosov Moscow State University, Mechanics and Mathematics Department, \\ Leninskie Gory 1, Moscow, 119234 Russia \\ ** National Research Nuclear University MEPhI, \\ 31 Kashirskoe Shosse, Moscow, 115409 Russia \\ ${ }^{* * *}$ Moscow Institute of Physics and Technology (National Research University), \\ Dolgoprudny, Moscow Region, 141700 Russia \\ E-mail: ${ }^{1}$ Aksenov.AV@gmail.com, ${ }^{2}$ Konstantin.Druzhkov@gmail.com
}

\begin{abstract}
A relation between variational principles for equations of continuum mechanics in Eulerian and Lagrangian descriptions is considered. It is shown that for a system of differential equations in Eulerian variables corresponding Lagrangian description is related to introducing nonlocal variables. The connection between these descriptions is obtained in terms of differential coverings. The relation between variational principles of a system of equations and its symplectic structures is discussed. It is shown that if a system of equations in Lagrangian variables can be derived from a variational principle then there is no corresponding variational principle in Eulerian variables.
\end{abstract}

\section{INTRODUCTION}

In continuum mechanics is well known, that for a given system of equations in Eulerian variables there exists natural analogue in Lagrangian variables, which describes the same continuum motion. The connection between the initial system and its analogue in Lagrangian variables is given by a differential covering [1]. From the Eulerian point of view, Lagrangian description contains nonlocal variables, which can affect on all main geometrical structures, including symmetries, conservation laws (see e.g. [2]), symplectic and Hamiltonian structures as well.

There are different kinds of variational principles in continuum mechanics (see e.g. [3]). In our paper, we shall mean a stationary-action principle by a variational principle. Action functional corresponds to some differential form (Lagrangian) $L$

$$
\mathcal{L}=\int L
$$

and its stationary points are solutions of the corresponding Euler-Lagrange equation $\mathrm{E}(L)=$ 0 , where $\mathrm{E}$ is the Euler operator.

The paper [4] is also devoted to the problem of connection between variational principles in Eulerian and Lagrangian descriptions. In this paper the authors used nonlocal variables and hence they deal with some intermediate description instead of purely Eulerian one. In our paper we obtain the relation between variational principles in Eulerian description (without nonlocal variables) and Lagrangian one. The relation is based on the concept of a symplectic structure for a system of differential equations. 


\section{LAGRANGIAN DESCRIPTION AS DIFFERENTIAL COVERING}

Let us consider the mass conservation law in continuum mechanics

$$
\rho_{t}+(u \rho)_{x}+(v \rho)_{y}+(w \rho)_{z}=0 .
$$

Here $\mathbf{v}=u \partial_{x}+v \partial_{y}+w \partial_{z}$ is a velocity field, $\rho$ is a mass density. Further we assume that $\rho>0$.

Choosing suitable nonlocal variables, one can introduce a potential for the mass conservation law, which satisfy the following relation

$$
\begin{aligned}
\rho d x \wedge d y \wedge d z-u \rho d t & \wedge d y \wedge d z+v \rho d t \wedge d x \wedge d z- \\
-w \rho d t & \wedge d x \wedge d y=d\left(\xi^{1} d \xi^{2} \wedge d \xi^{3}\right)=d \xi^{1} \wedge d \xi^{2} \wedge d \xi^{3} .
\end{aligned}
$$

The relation (2) is equivalent to the following system of equations

$$
\rho=\operatorname{det}\left(\frac{\partial \xi}{\partial x}\right), \quad \xi_{t}^{i}+u \xi_{x}^{i}+v \xi_{y}^{i}+w \xi_{z}^{i}=0, \quad i=1,2,3 .
$$

Here by $\partial \xi / \partial x$ we denote the corresponding Jacobi matrix. Note that such functions $\xi^{i}(t, x, y, z)$ are Lagrangian variables.

Remark 1. Transformations that preserve the volume form in $\left(\xi^{1}, \xi^{2}, \xi^{3}\right)$-space form symmetry group for equations in Lagrangian variables.

Since the only consistency condition for the system (3) is the mass conservation law (1), then for any system of equations in Eulerian variables the potential (2) determines its differential covering. If a system of equations at hand is of the form (in Eulerian variables)

$$
F^{1}=0, \quad \ldots, \quad F^{m}=0,
$$

then the mass conservation law allows us to derive the covering system in the following form

$$
\begin{aligned}
& F^{1}=0, \quad \ldots, \quad F^{m}=0, \\
& \rho=\operatorname{det}\left(\frac{\partial \xi}{\partial x}\right), \quad \xi_{t}^{i}+u \xi_{x}^{i}+v \xi_{y}^{i}+w \xi_{z}^{i}=0, \quad i=1,2,3 .
\end{aligned}
$$

One can choose $x, y, z$ as new dependent variables for (5) and obtain usual Lagrangian representation of the system (4). Therefore, for a system of equations in Eulerian variables the corresponding Lagrangian description can be considered as differential covering. This fact allows to lift some geometrical structures from the Eulerian description to the Lagrangian one.

Remark 2. Other conservation laws of systems of equations also allow to introduce nonlocal variables and to obtain differential coverings.

\section{INFINITE PROLONGATION OF A SYSTEM OF DIFFERENTIAL EQUATIONS}

Lets consider a system of differential equations

$$
F^{1}=0, \quad \ldots, \quad F^{m}=0,
$$


where $F^{i}$ are functions of independent variables $x^{1}, \ldots, x^{n}$, dependent variables $u^{1}, \ldots, u^{m}$ and derivatives up to some finite order. Denote multi-index of the form $\alpha_{i} x^{i}$ by $\alpha$. Here and further we assume summation over repeated indices. Put

$$
D_{\alpha}=D_{x^{1}}^{\alpha_{1}} \circ \ldots \circ D_{x^{n}}^{\alpha_{n}}, \quad u_{\alpha}^{i}=D_{\alpha}\left(u^{i}\right)
$$

where $D_{x^{i}}$ are the operators of total derivatives. Denote the infinite prolongation of the system of equations $F=0$ by $\mathcal{E}$

$$
\mathcal{E}: \quad F^{i}=0, \quad D_{x^{1}}\left(F^{i}\right)=0, \quad D_{x^{2}}\left(F^{i}\right)=0, \quad \ldots
$$

The universal linearization operator [1] for $F$ denote by $l_{F}$. It acts on a vector-function $\varphi=\left(\varphi^{1}, \ldots, \varphi^{m}\right)^{T}$ by the formula

$$
\left(l_{F}(\varphi)\right)^{i}=l_{F j}^{i}\left(\varphi^{j}\right)=\frac{\partial F^{i}}{\partial u_{\alpha}^{j}} D_{\alpha}\left(\varphi^{j}\right) .
$$

Denote by $l_{\mathcal{E}}$ the restriction of the operator $l_{F}$ to the system $\mathcal{E}$.

Further we deal with $l$-normal [1] systems only. We shall say that a system of equations of the form

$$
u_{b_{1} x^{n}}^{1}=\Phi^{1}, \quad \ldots, \quad u_{b_{m} x^{n}}^{m}=\Phi^{m}
$$

has the extended Kovalevskaya form if all $b_{i}$ are positive integers and the right-hand side $\left(\Phi^{1}, \ldots, \Phi^{m}\right)^{T}$ is independent of the variables $u_{b_{i} x^{n}}^{i}$ and their derivatives. Systems of equations, which can be written in an extended Kovalevskaya form, are l-normal. Most systems of equations in continuum mechanics can be written in an extended Kovalevskaya form.

Example 1. In dimensionless variables the Navier-Stokes system of equations for incompressible fluid

$$
\begin{aligned}
& u_{t}+u u_{x}+v u_{y}+w u_{z}=-p_{x}+u_{x x}+u_{y y}+u_{z z}, \\
& v_{t}+u v_{x}+v v_{y}+w v_{z}=-p_{y}+v_{x x}+v_{y y}+v_{z z}, \\
& w_{t}+u w_{x}+v w_{y}+w w_{z}=-p_{z}+w_{x x}+w_{y y}+w_{z z}, \\
& u_{x}+v_{y}+w_{z}=0
\end{aligned}
$$

can be written in the extended Kovalevskaya form for $x^{n}=z, b=(2,2,1,1)$. One can eliminate $w_{z}$ from the fourth equation; eliminate $u_{z z}$ from the first equation; eliminate $v_{z z}$ from the second equation; eliminate $p_{z}$ from the third equation. The Euler system of equations also can be written in an extended Kovalevskaya form.

\section{SYMPLECTIC STRUCTURES AND VARIATIONAL PRINCIPLES}

For $l$-normal systems of differential equations, similar to the classical differential geometry, there are two equivalent representations of symplectic structures: as equivalence classes of differential forms, and as equivalence classes of operators in total derivatives (see, e.g. [1]). 1. A symplectic structure of a system of equations $\mathcal{E}$ is a closed variational 2-form on $\mathcal{E}$, i.e. an element of the kernel of the variational differential

$$
\delta: E_{1}^{2, n-1}(\mathcal{E}) \rightarrow E_{1}^{3, n-1}(\mathcal{E})
$$


Here $E_{1}^{p, n-1}(\mathcal{E})$ are groups of variational $p$-forms from the $\mathcal{C}$-spectral sequence.

2. Variational 2-forms of a system $\mathcal{E}$ can be described as operators in total derivatives $\Delta$, which satisfy the relation

$$
\Delta^{*} \circ l_{\mathcal{E}}=l_{\mathcal{E}}^{*} \circ \Delta
$$

modulo operators of the form $\nabla \circ l_{\mathcal{E}}$, where $\nabla=\nabla^{*}$. Here the operator $\Delta^{*}$ is formally adjoint to an operator $\Delta$.

Each symplectic structure of an $l$-normal system of differential equations determines a map from its symmetries to variational 1-forms (i.e. it determines a Noether theorem). This map can be equivalently described in both ways: in terms of variational forms and in terms of operators. Such operators map symmetries of $\mathcal{E}$ to $\operatorname{Ker} l_{\mathcal{E}}^{*}$, which is isomorphic to the group of variational 1 -forms $E_{1}^{1, n-1}(\mathcal{E})$ in $l$-normal case.

Remark 3. The definition of a symplectic structure as a closed variational 2-form allows us to lift symplectic structures in coverings.

We shall say that a system of equations $\mathcal{E}$ is variational if there exists a Lagrangian $L$, such that the following relation holds

$$
F=\mathrm{E}(L)
$$

where $\mathrm{E}$ is the Euler operator. Also we shall say that the system of equations $\mathcal{E}$ admits variational principle if for some operator in total derivatives $A$ exists a Lagrangian $L$, such that the following relation holds

$$
A(F)=\mathrm{E}(L) .
$$

In such situation the operator $\Delta=\left.A^{*}\right|_{\mathcal{E}}$ determines the symplectic structure for the corresponding system $\mathcal{E}$. Let us say that a symplectic structure of $\mathcal{E}$, which can be obtained in this way, is related to a variational principle.

\section{MAIN RESULTS}

Informally speaking, symplectic structures of a system of differential equations can be considered as its "Noether theorems". Therefore it is quite natural to expect that at least for an $l$-normal system of differential equations its symplectic structures can be used for deriving variational principles.

Theorem 1. If an l-normal system of equations $\mathcal{E}$ has trivial de Rham cohomology group $H^{n+1}(\mathcal{E})$, then each symplectic structure of $\mathcal{E}$ is related to a variational principle.

Proof. Each symplectic structure can be considered as equivalence class of differential forms. As it follows from [5] a symplectic structure $\omega \in E_{1}^{2, n-1}(\mathcal{E})$ is related to a variational principle if and only if it is generated by an exact differential form (as equivalence class of differential forms). Each closed variational 2-form $\omega$ either belongs to $\delta E_{1}^{1, n-1}(\mathcal{E})$ or generates nontrivial element $[\omega]$ of cohomology group $E_{2}^{2, n-1}(\mathcal{E})$. If $\omega \in \delta E_{1}^{1, n-1}(\mathcal{E})$, then it is generated by an exact differential form. Assume now that $[\omega] \neq 0$. According to the two-line theorem (see [1]) group $E_{2}^{4, n-2}(\mathcal{E})$ is trivial, hence $[\omega]$ is a cocycle. The group $E_{3}^{2, n-1}(\mathcal{E})$ is also trivial, then $[\omega] \in \operatorname{Im} d_{2}^{0, n}$, where

$$
d_{2}^{0, n}: E_{2}^{0, n}(\mathcal{E}) \rightarrow E_{2}^{2, n-1}(\mathcal{E})
$$


Then $[\omega]=\omega+\delta E_{1}^{1, n-1}(\mathcal{E})$ is generated by an exact differential form. Hence symplectic structure $\omega$ is still generated by an exact differential form and is related to a variational principle.

A system of equations in an extended Kovalevskaya form admits a canonical way to derive a variational principle from a symplectic structure [6]. Therefore, if a system of equations is written in an extended Kovalevskaya form in both Eulerian and Lagrangian descriptions, then the relation between variational principles in these descriptions can be obtained in terms of symplectic structures. Each variational principle in Eulerian description generates unique symplectic structure, which can be lifted to the Lagrangian description. The last step is to derive the corresponding variational principle from this lift.

Let $F=0$ be a system of differential equations in Eulerian variables, $\mathcal{E}$ be its infinite prolongation, $\widetilde{\mathcal{E}}$ be the corresponding system in Lagrangian variables. Denote the covering from $\widetilde{\mathcal{E}}$ to $\mathcal{E}$ by $\tau$.

Theorem 2. If the system of equations $\widetilde{\mathcal{E}}$ is l-normal variational system, then the corresponding symplectic structure of $\widetilde{\mathcal{E}}$ is not a lift of a symplectic structure of $\mathcal{E}$.

Proof. Consider algebra $\tau_{*}$-sym $\widetilde{\mathcal{E}}$ of $\tau$-projectable symmetries of $\widetilde{\mathcal{E}}$. Then for any variational 2-form $\omega \in E_{1}^{2, n-1}(\mathcal{E})$ the following diagram is commutative

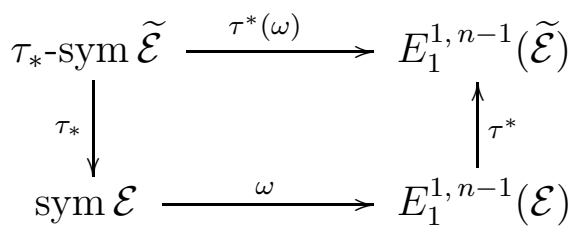

Thus, for a symmetry $\varphi \in \tau_{*}$-sym $\widetilde{\mathcal{E}}$, which acts in a fibre of $\tau$, holds the relation $\tau^{*}(\omega)(\varphi)=0$, i.e. the lift of a symplectic structure of $\mathcal{E}$ is degenerate and the equivalence class of operators, which corresponds to $\tau^{*}(\omega)$, can not contain the identical operator.

This theorem shows that if an $l$-normal system of equations in Lagrangian variables is variational, then the corresponding variational principle has no analogues in Eulerian variables. Similar result holds true for any covering from an $l$-normal system of differential equations, such that the fiber symmetry algebra is nontrivial. In particular, it holds true for coverings from $l$-normal systems of differential equations, which based on introduction of potentials for conservation laws.

\section{EXAMPLES}

Lets consider two examples of variational $l$-normal systems in Lagrangian variables.

Example 2. Equations of motion a polytropic gas $\left(p=C \rho^{\gamma}\right)$ in Lagrangian variables are Euler-Lagrange equations for Lagrangian

$$
L=\left(\frac{x_{t}^{2}+y_{t}^{2}+z_{t}^{2}}{2}-V-U\right) d t \wedge d \xi^{1} \wedge d \xi^{2} \wedge d \xi^{3},
$$

where $V$ is a potential energy, $U$ is an internal energy. The corresponding system of equations can be written in the extended Kovalevskaya form

$$
x_{t t}=-\frac{\delta(V+U)}{\delta x}, \quad y_{t t}=-\frac{\delta(V+U)}{\delta y}, \quad z_{t t}=-\frac{\delta(V+U)}{\delta z} .
$$


Thus, this system of equations is $l$-normal and there is no corresponding variational principle for equations of motion a polytropic gas in (purely) Eulerian variables.

Example 3. The Green-Naghdi equation in Lagrangian variables can be derived from variational principle for Lagrangian [7]

$$
\begin{aligned}
L=( & \frac{x_{t}^{2}}{2}\left(1+\varepsilon\left(H^{\prime \prime 2}+\frac{x_{m m}}{x_{m}^{3}} H^{\prime \prime}-\frac{1}{2 x_{m}} H^{\prime \prime \prime}\right)\right)+ \\
& \left.+\frac{1}{6 x_{m}^{4}}\left(\varepsilon x_{t m}^{2}-3 g x_{m}^{2}\left(2 H x_{m m}+x_{m}\right)\right)\right) d t \wedge d m
\end{aligned}
$$

where function $H^{\prime}(x)$ describes the bottom topography, $g$ is the gravitational acceleration, $\varepsilon$ is a small parameter. After $\pi / 4$-rotation in $(t, m)$-space the corresponding equation can be written in the extended Kovalevskaya form. Thus, this equation is also $l$-normal and there is no corresponding variational principle for the Green-Naghdi equations in Eulerian variables.

\section{CONCLUSION}

The construction of a symplectic structure for a system of differential equations allowed to connect variational principles in Eulerian and Lagrangian variables. The connection is based on the fact that one can consider Lagrangian variables as nonlocal variables in a differential covering. Besides, the obtained result allowed to show that a nondegenerate variational principle in Lagrangian variables is not related to any variational principle in Eulerian variables. However, each variational principle in Eulerian variables is related to some variational principle in Lagrangian variables. Thus, from this point of view the Lagrangian description is preferred over the Eulerian one.

\section{REFERENCES}

1. A.V. Bocharov, V.N. Chetverikov, S.V. Duzhin, N.G. Khor'kova, I.S. Krasil'shchik, A.V. Samokhin, Yu.N. Torkhov, A.M. Verbovetsky, and A.M. Vinogradov, Symmetries and conservation laws for differential equations of mathematical physics, American Mathematical Society, 182 (1999).

2. A.V. Aksenov, K.P. Druzhkov, "Conservation laws of the equation of one-dimensional shallow water over uneven bottom in Lagrange's variables," International Journal of NonLinear Mechanics 119, 103348 (2020).

3. V.L. Berdichevsky, Variational Principles of Continuum Mechanics. I. Fundamentals (Springer-Verlag Berlin Heidelberg, 2009).

4. F. Bampi, A. Morro, "The connection between variational principles in Eulerian and Lagrangian descriptions," Journal of Mathematical Physics 25, 2418 (1984).

5. I. Khavkine, "Presymplectic current and the inverse problem of the calculus of variations," Journal of Mathematical Physics 54, 111502 (2013) .

6. K.P. Druzhkov, "Extendable symplectic structures and the inverse problem of the calculus of variations for systems of equations written in generalized Kovalevskaya form," Journal of Geometry and Physics 161, 104013 (2021). 
7. V.A. Dorodnitsyn, E.I. Kaptsov, and S.V. Meleshko, "Symmetries, Conservation Laws, Invariant Solutions and Difference Schemes of the One-dimensional Green-Naghdi Equations," Journal of Nonlinear Mathematical Physics 28, 90-107 (2021). 